\title{
TOLERANCE SYNTHESIS OF MECHANISMS: A ROBUST DESIGN APPROACH
}

\author{
Stéphane Caro, Fouad Bennis, Philippe Wenger \\ Institut de Recherche en Communications et Cybernétique de Nantes * \\ 1, rue de la Noë, 44321 Nantes, France \\ Stephane.Caro@irccyn.ec-nantes.fr \\ Fouad.Bennis@irccyn.ec-nantes.fr \\ Philippe.Wenger@irccyn.ec-nantes.fr
}

\begin{abstract}
This paper provides a new robust design method to dimension a mechanism and to synthesize its dimensional tolerances. The general issue is to find a robust mechanism for a given task, and to compute its optimal dimensional tolerances. For that purpose, the developed approach follows two consecutive steps, which are independent and complementary. First, the dimensions of the mechanism are computed by means of an appropriate robustness index, which is used to minimize the sensitivity of its performances to variations. These robust dimensions are obtained independently of the amount of variations, and tolerate globally the largest variations. Thus, knowing the acceptable performance error of the mechanism, the second step aims at computing the optimal dimensional tolerances of the mechanism by means of the new tolerance synthesis method. This method is used to find the best distribution of the error between the dimensions of the mechanism. Two serial manipulators are studied to illustrate the theory.
\end{abstract}

Keywords: robust design, tolerance synthesis, mechanism, variations, sensitivity ellipsoid, optimization.

\section{Introduction}

Every engineering design is subject to variations that can arise from a variety of sources, including manufacturing operations, variations in material properties, and the operating environment. When variations are ignored, nonrobust designs can result, which are expensive to produce or fail in service. Besides, the robustness of a mechanism is important when calibration is necessary because the lower the sensitivity of the mechanism to dimensional variations, the easier its calibration [1].

*IRCCyN: UMR n 6597 CNRS, École Centrale de Nantes, Université de Nantes, École des Mines de Nantes
The concept of robust design may be first used by Taguchi. He introduced the concept of parameter design to improve the quality of a product whose manufacturing process involves significant variability or noise [2]. Robust design aims at minimizing the sensitivity of performances to variations without controlling the causes of these variations. In the last decades, several authors have contributed to the formulation and the improvement of robust design problems. Kalsi et al. [3] introduced a technique to reduce the effects of uncertainty and incorporate flexibility in the design of complex engineering systems involving multiple decision-makers. Chen et al. [ studied two broad categories of problems namely, (i) Type 1: minimizing variations in performance caused by variations in noise factors (uncontrollable parameters) and (ii) Type 2: minimizing variations in performance caused by variations in control factors (design variables, $D V$ ). Sundaresan et al. [5] developed a procedure incorporating uncertainties in $D V$ and variations in constraints due to these uncertainties.

The dimensional tolerances of a mechanism are fixed according to various parameters such as the manufacturing process, the performance tolerances, the manufacturing cost. Chase et al. [6,7] presented the Direct Linearization Method for tolerance analysis of 2-D and 3-D mechanical assemblies. Parkinson [8] used a deterministic method of robust design to determine the optimum nominal dimensions of an assembly in order to improve the assembly quality without tightening tolerances. Moreover, Rajagopalan and Cutkosky [9] used similar methods to analyse the performance errors of mechanisms fabricated in-Situ.

Some optimization methods for tolerance synthesis exist in the literature. Zhang and Wang [10] used a simulated annealing algorithm to maximize the robustness of product by appropriately allocating assembly and machining tolerances. Lee et al. [11] presented a tolerance synthesis method for nonlinear sys- 
tems based on nonlinear programming, whereas Gadallah and ElMaraghy [12] presented a method using a system of experimental design. Zhu and Ting [13] used the theory of performance sensitivity distribution to study the sensitivity of the system to variations, and selected one manipulator among six, by means of a robustness index. They defined the tolerance box as a contraction of the circumscribe box of the design sensitivity ellipsoid of the mechanism. The link between dimensional tolerances and product's cost is presented in several works [14].

The paper focuses on mechanisms, which are assemblies of moving parts performing a complete functional motion.

Here, the study of a mechanism and the calculation of its dimensional tolerances are conducted in two consecutive steps. First, its dimensions are computed by means of a robustness index, which is proposed to minimize the sensitivity of its performances to variations. For example, a robust dimensioning of the $2 R$ manipulator, whose end effector $E$ has to hit point $P$ with the highest precision, is depicted in Fig. 1. For this dimensioning, the links of the manipulator are perpendicular when $E$ is supposed to hit $P$. In this configuration, the maximum positioning error of $E$, $\varepsilon$, due to dimensional variations, is a minimum.

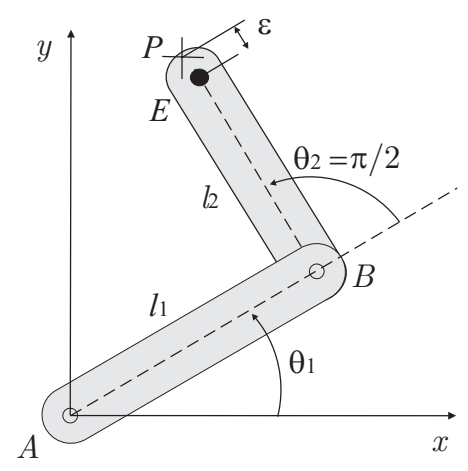

Figure 1. A Robust Dimensioning of the $2 R$ Manipulator

Then, knowing the acceptable performance error of the mechanism, the second step aims at computing the optimal dimensional tolerances of the mechanism by means of a new tolerance synthesis method. This method is based on the robustness approach of the first step.

The formulation of a robust design problem is given in section 2 Section 3 discusses of an appropriate robustness index for mechanisms. The new tolerance synthesis method is developed in section 4. Finally, a $2 R$ manipulator and a $3 R$ manipulator are studied in section 5 to illustrate the theory.

\section{Robust design problem}

In a robust design problem, the distinction is made between three sets: (i) the set of design variables $(D V)$ whose nominal values can be selected between the range of upper and lower bounds, they are controllable; (ii) the set of design parameters $(D P)$ that cannot be adjusted by the designer, they are uncontrollable; (iii) the set of performance functions. The $l$-dimensional vector of design variables is denoted by $\mathbf{x}=\left[\begin{array}{lll}x_{1} & x_{2} \cdots x_{l}\end{array}\right]^{T}$. The $m$-dimensional vector of design parameters is denoted by $\mathbf{p}=$ $\left[\begin{array}{ll}p_{1} & p_{2} \\ \cdots & p_{m}\end{array}\right]^{T}$. Performance functions are grouped into the $n$ dimensional vector $\mathbf{f}=\left[\begin{array}{lll}f_{1} & f_{2} \cdots f_{n}\end{array}\right]^{T}$, [15]. $D V$ are, however, subject to uncontrollable variations because of manufacturing errors, wear, or other uncertainties, although their nominal value is fixed.

For instance, for the slider-crank mechanism depicted by Fig. 2. $\mathbf{f}=\left\langle N>, \mathbf{x}=\left[\begin{array}{lll}l_{c} & l_{r} & e\end{array}\right]^{T}\right.$, and $\mathbf{p}=\left[\begin{array}{ll}f_{p} & \mu\end{array}\right]$ where $\langle N\rangle$ is the average side force on piston to be minimized. $l_{c}$ and $l_{r}$ are the lengths of the rod and the crank of the mechanism. $e$ is the eccentricity between the crank and the piston. $f_{p}$ is the force on piston and $\mu$ is the friction coefficient between the piston and the cylinder .

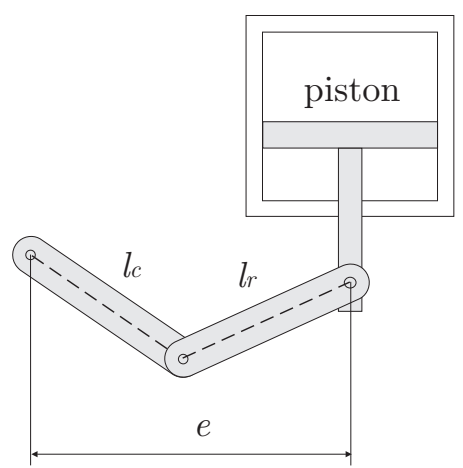

Figure 2. Slider-Crank Mechanism

A system is robust when its performance is as little sensitive as possible to variations. Performance function $\mathbf{f}$ depends on $D V$ and $D P$, which are supposed to be independent.

$$
\mathbf{f}=\mathbf{f}(\mathbf{x}, \mathbf{p})
$$

Here, the study of the sensitivity of the system to variations is based on the theory of performance sensitivity distribution.

$$
\delta \mathbf{f}=\left[\mathbf{J}_{x} \mathbf{J}_{p}\right]\left[\delta \mathbf{x}^{T} \delta \mathbf{p}^{T}\right]^{T}=\mathbf{J} \delta \mathbf{X}
$$


In this theory, a Jacobian matrix $\mathbf{J}$ describes the effect of the component variations to the system performance, as depicted by eq. (2) where $\mathbf{J}_{x}=\partial \mathbf{f} / \partial \mathbf{x}, \mathbf{J}_{p}=\partial \mathbf{f} / \partial \mathbf{p}, \mathbf{J}=\left[\mathbf{J}_{x} \mathbf{J}_{p}\right], \mathbf{X}^{T}=\left[\mathbf{x}^{T} \mathbf{p}^{T}\right]$. $\delta \mathbf{x}$ and $\delta \mathbf{p}$ are the variations in $D V$ and in $D P$, respectively. $\mathbf{J}_{x}$ and $\mathbf{J}_{p}$ are the $(n \times l)$ sensitivity Jacobian matrix of $\mathbf{f}$ with respect to $\mathbf{x}$ and the $(n \times m)$ sensitivity Jacobian matrix of $\mathbf{f}$ with respect to $\mathbf{p}$, respectively. If variations in $D V$ are not taken into account, then $\mathbf{J}=\mathbf{J}_{p}$ and $\mathbf{X}=\mathbf{p}$. On the contrary, $\mathbf{J}=\mathbf{J}_{x}$ and $\mathbf{X}=\mathbf{x}$ when only variations in $D V$ are considered.

The performance distribution is characterized in the variation space by a set of eigenvalues and eigenvectors, i.e.: by a hyper-ellipsoid. Without loss of generality, assuming that variations in $D V$ are negligible and that there are only two $D P$, this design sensitivity hyper-ellipsoid is an ellipse depicted in Fig. 3 . $\sigma_{1}$ and $\sigma_{2}$ are the smallest and the largest singular values of $\mathbf{J}$, respectively, and $\mathbf{q}_{1}, \mathbf{q}_{2}$ are their corresponding eigenvectors. Lengths of semi-axes are inversely proportional to singular values of $\mathbf{J}$. Points on the ellipse surface lead to the same norm of performance variation, $\|\delta \mathbf{f}\|_{2}$, where $\|.\|_{2}$ depicts the Euclidean norm. Moreover, the performance is the least sensitive to variations in the direction of $\mathbf{q}_{1}$ and the most sensitive to variations in the direction of $\mathbf{q}_{2}$.

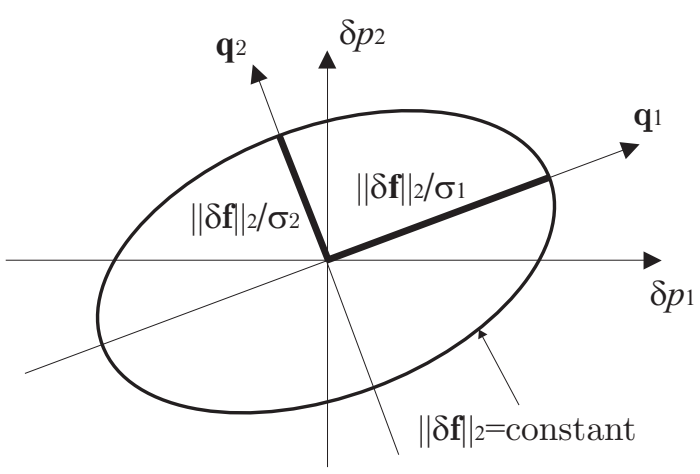

Figure 3. Design Sensitivity Ellipsoid

A mechanism is robust when the sensitivity $S$ of its performances to variations is a minimum. Therefore, $S$ can be defined as the ratio of the Euclidean norm of variations in its performances, $\|\delta \mathbf{f}\|_{2}$, and the Euclidean norm of variations in $D V$ and $D P,\|\delta \mathbf{X}\|_{2},[15]$ ]. $S$ represents a variation transmission ratio and means the amount of variations transmitted from the sources to the design. Besides, eq.(3) follows from eq.(2) and means that $S$ is bounded by the smallest singular value, $\sigma_{\min }$, and the largest singular value, $\sigma_{\max }$, of sensitivity Jacobian matrix $\mathbf{J}$.

$$
\sigma_{\min } \leq S=\frac{\|\delta \mathbf{f}\|_{2}}{\|\delta \mathbf{X}\|_{2}} \leq \sigma_{\max }
$$

\section{Choice of an appropriate robustness index}

In order to obtain a robust solution independently of the amount of variations in $D V$ and $D P$, a judicious robustness index is required. The robustness indices usually found in the recent literature are the condition number and the Euclidean norm of the sensitivity Jacobian matrix, J. Al-Widyan and Angeles [15], Ting and Long [16] used the condition number of $\mathbf{J}$. Zhu [13] and $\mathrm{Hu}$ et al. [17] suggested the use of the Euclidean norm of $\mathbf{J}$. In this section, it is shown that the Euclidean norm of $\mathbf{J}$ is more appropriate for the robust design of mechanisms.

The condition number of a matrix is the ratio of its largest singular value to its smallest singular value. Let $R I_{1}$ be the condition number of $\mathbf{J}$.

$$
R I_{1}=\|\mathbf{J}\|_{2}\left\|\mathbf{J}^{-1}\right\|_{2}=\frac{\sigma_{\max }}{\sigma_{\min }}
$$

According to [16, 15], a design is robust when $R I_{1}$ is a minimum. Assuming that only variations in $D P$ are considered, each variation $\delta p_{i}$ has the same influence on the norm of variations in performance when $R I_{1}$ is unitary, i.e.: the sensitivity ellipsoid is a sphere. Although this property is interesting, the previous index is not sufficient because the influence of $\delta p_{i}$ on performance is not necessarily a minimum when $R I_{1}$ is unitary, [18]. Indeed, the condition number, $R I_{1}$, can be small even if the values of $\sigma_{\min }$ and $\sigma_{\text {max }}$ are large.

A singular value of $\mathbf{J}$ corresponds to the error transmission factor in the direction of its corresponding eigenvector and in the space of variations. The ideal solution is the minimization of all the singular values of $\mathbf{J}$, but is not easy to obtain. According to eq.(3), a compromise solution is to minimize the upper bound of $S$, which is the largest singular value of $\mathbf{J}$. Thus, a second robustness index, $R I_{2}$, is defined by eq.(5).

$$
R I_{2}=\|\mathbf{J}\|_{2}=\sigma_{\max }
$$

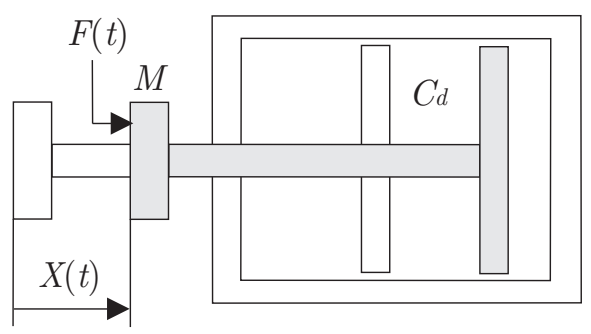

Figure 4. Damper 
The damper shown in Fig. 4 is studied to compare $R I_{1}$ and $R I_{2}$. The design variables are mass $M$ and damping coefficient $C_{d}$ to be determined with the aim of keeping the magnitude of displacement $X_{0}$ at a nominal value of $0.1 \mathrm{~m}$, while the magnitude $F_{0}$ of the excitation force $F(t)=F_{0} \cos (\omega t)$ and its pulsation $\omega$ undergo considerable variations beyond the control of the designer: $F_{0}=10 \mathrm{~N}, \omega=2 \pi \mathrm{rad} / \mathrm{s}$. The displacement is equal to $X(t)=X_{0} \cos (\omega t+\phi)$ where $\phi$ is the phase. Moreover, the following relations exist:

$$
X_{0}=\frac{F_{0}}{\omega \sqrt{C_{d}^{2}+\omega^{2} M^{2}}}, \quad \phi=\tan ^{-1}\left(\frac{\omega M}{C_{d}}\right)
$$

$\mathbf{x}=\left[M C_{d}\right]^{T}, \mathbf{p}=\left[F_{0} \omega\right]^{T}, \mathbf{f}=\left[X_{0} \phi\right]^{T}$. Equation (6) gives the relation between variations in $\mathbf{f}$ and variations in $\mathbf{p}$.

$$
\delta \mathbf{f}=\mathbf{J} \delta \mathbf{p}
$$

where the sensitivity Jacobian matrix depends on $\mathbf{x}$ and $\mathbf{p}$, and is equal to:

$$
\mathbf{J}=\mathbf{J}_{p}=\left[\begin{array}{cc}
1 & -1-\alpha^{2} \\
0 & \alpha \sqrt{1-\alpha^{2}}
\end{array}\right], \quad \alpha=\frac{X_{0}}{F_{0}} \omega^{2} M
$$

and

$$
\delta \mathbf{f}=\left[\begin{array}{c}
\delta X_{0} / X_{0} \\
\delta \phi
\end{array}\right], \quad \delta \mathbf{p}=\left[\begin{array}{c}
\delta F_{0} / F_{0} \\
\delta \omega / \omega
\end{array}\right]
$$

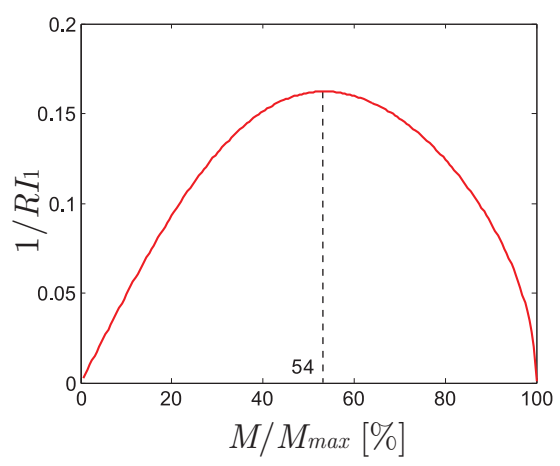

Figure 5. Robustness index $R I_{1}$

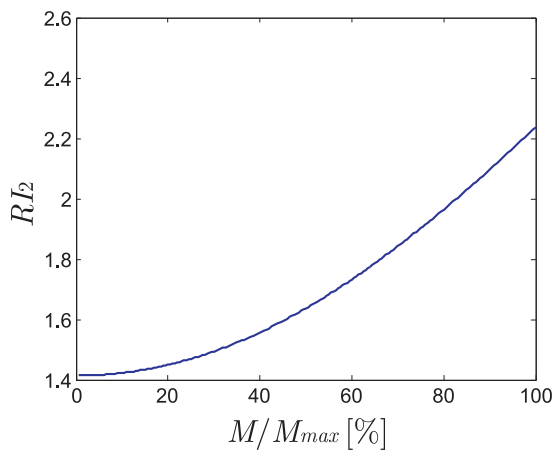

Figure 6. Robustness index $R I_{2}$

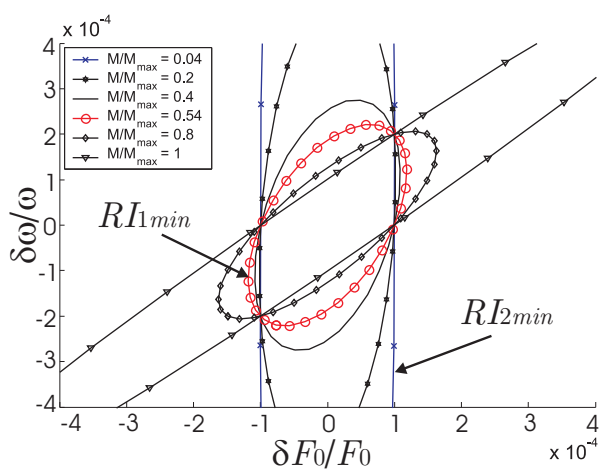

Figure 7. Design Sensitivity Ellipses

Figures 5, 6 depict robustness indices $R I_{1}$ and $R I_{2}$ of the damper with respect to $M$, respectively. In Fig. $R I_{1}$ is a minimum when $M / M_{\max }=0.54$ with $M_{\max }=2.533 \mathrm{~kg}$. Fig.6 shows that $R I_{2}$ increases with $M$. According to Fig.7, which depicts some design sensitivity ellipses of the damper, plotted for different values of $M$, the more $M$ tends towards zero, the larger the size of the ellipse. It means that the design can tolerate globally more variations in $F_{0}$ and $\omega$, i.e.: it is robust. Besides, the ellipse corresponding to the value of $M / M_{\max }$ that minimises $R I_{1}$ is the smallest one. Therefore, minimizing $R I_{1}$ is not equivalent to minimizing the influence of variations in $D P$ on performance function.

Chen et al. [4] and Parkinson [19] proposed an optimization algorithm to increase the robustness of a design without using robustness indices. However, they need to know the magnitude of source variations to use their algorithm. Assuming that $\Delta F_{0} / F_{0}=0.1, \Delta \omega / \omega=0.1$ and $M / M_{\max } \geq 1 / 2$, their algorithm converges on $M / M_{\max }=1 / 2$ and $C_{d}=13.78 \mathrm{~N} . \mathrm{s.m}{ }^{-1}$ in order to minimize variations in $X_{0}$ and $\phi$. So, according to [丹] and [19], the mass of the damper is minimized to make it robust, like with robustness index $R I_{2}$. In conclusion, the study of the damper confirms that $R I_{2}$ is more suitable than $R I_{1}$ to evaluate the robustness 
of a mechanism. $R I_{2}$ is used in the following sections of this paper.

Remark: It is important not to confuse symbols $\delta$ and $\Delta$. $\delta v$ depicts the variation of variable $v$ and $\Delta v$ depicts its tolerance, i.e.: $-\Delta v \leq \delta v \leq \Delta v$.

\section{An efficient tolerance synthesis method}

The dimensional tolerances of a mechanism are usually fixed according to various parameters such as the manufacturing process, the performance tolerances, the manufacturing cost. Some optimization methods for tolerance synthesis exist in the literature. Zhu and Ting [13] defined the tolerance box as a contraction of the circumscribe box of the design sensitivity ellipsoid of the mechanism. However, this Tolerance Box, called Zhu-TB, includes some rejects, cf Fig 8 .

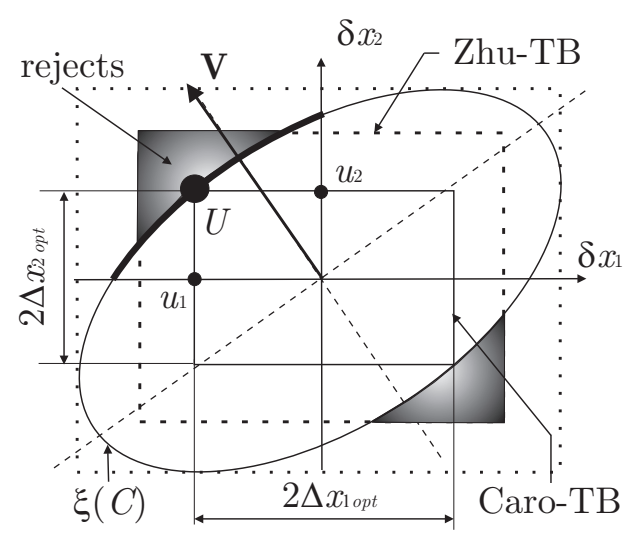

Figure 8. Tolerance Synthesis, $l=2$

Some works in the literature deal with the link between dimensional tolerances and product's cost [14, 9]. Here, the cost of a mechanism is supposed to decrease when its dimensional tolerances increase. Thus, a new tolerance synthesis method is proposed, which aims at finding the largest tolerance box of a mechanism that does not include rejects. Let $\xi(C)$ be the design sensitivity ellipsoid of a mechanism corresponding to a norm of variations in its performance equal to $C$. Assuming that this norm has to be smaller than $C$, the optimal tolerance box is supposed to be the largest box included in $\xi(C)$. This tolerance box called Caro-TB and depicted in Fig. 8, is smaller than Zhu-TB, but does not include any reject. The choice of the tolerance box depends on the wish of the designer. However, it is always important to know the solution without rejects because the cost of the loss due to rejects can be estimated from this solution.

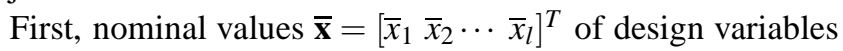
are computed from robustness index $R I_{2}$, presented in section 3 .
Then, their optimal dimensional tolerances $\Delta x_{i o p t}$ are computed using the following optimization algorithm:

$$
\begin{aligned}
& \left\{\begin{aligned}
& \max _{\mathbf{u}} \prod_{i=1}^{l}\left|u_{i}\right| \\
& \text { s.t. } U\left(u_{1}, u_{2}, \cdots, u_{l}\right) \in \xi(C) \\
& u_{i} \cdot \operatorname{sign}\left(V_{i}\right) \geq 0, i=1, \cdots, l \\
&\left|u_{i}\right| \geq \Delta x_{\text {imin }}, i=1, \cdots, l
\end{aligned}\right. \\
& \Delta x_{\text {iopt }}=\left|u_{i}\right|, \quad \bar{x}_{i}-\Delta x_{\text {iopt }} \leq x_{i} \leq \bar{x}_{i}+\Delta x_{\text {iopt }}, \quad i=1, \cdots, l
\end{aligned}
$$

This algorithm consists in maximizing the hyper-volume of the tolerance box included in $\xi(C)$. $\mathbf{V}$ is the eigenvector corresponding to the maximum singular value of the sensitivity Jacobian matrix of the mechanism and $V_{i}$ is its $i^{t h}$ component. Besides, point $U$ whose coordinates are $\left(u_{1}, u_{2} \cdots, u_{l}\right)$ belongs to $\xi(C)$ if and only if $\mathbf{u}^{T} \mathbf{J}_{x}^{T} \mathbf{J}_{x} \mathbf{u}=C^{2}$ where $\mathbf{u}=\left[u_{1}, u_{2}, \cdots, u_{l}\right]^{T}$. Moreover, each dimensional tolerance $\Delta x_{i}$ has to be higher than a minimum dimensional tolerance $\Delta x_{\text {imin }}$, depending on the manufacturing process and $\bar{x}_{i}$. For instance, Fig. 8 depicts all the possible positions of $U$ when $l=2$ and $V_{1}, V_{2}$ are negative and positive, respectively.

In the following, a $2 R$ and a $3 R$ manipulators are studied to illustrate the proposed robust design and tolerance synthesis methods. If the positioning error of the end effector has to be smaller than a scalar $C$ at $n$ different goal poses, it means that the tolerance box has to be included in $n$ design sensitivity ellipsoids because each pose of the manipulator is associated with a design sensitivity ellipsoid. However, the tolerance synthesis algorithm works with only one design sensitivity ellipsoid. To cope with this problem, we consider the most restrictive ellipsoid, $\xi_{m r}$. As for any serial manipulator, a unitary variation in one design variable and no variation in the others lead to a unitary positioning error of its end effector, the design sensitivity ellipsoids intersect at $2^{l}$ points where $l$ is the number of design variables. Therefore, $\xi_{m r}$ is the ellipsoid with the smallest small axis among the $n$ design sensitivity ellipsoids.

\section{Case studies}

\subsection{Study of a $2 R$ manipulator}

The mechanism studied in this section is a serial $2 R$ manipulator, depicted in Fig.9. It is composed of two revolute joints and two links $A B$ and $B E$ of lengths $l_{1}$ and $l_{2}$, respectively. First, the manipulator is designed, so that its end-effector $E$ can hit all points of a target $S_{T}$, and to be as little sensitive as possible to dimensional variations. Indeed, the lower the sensitivity of the manipulator to dimensional variations, the easier its calibration [1]]. Subsequently, the tolerance synthesis method introduced in section 4 is used to compute its optimal dimensional tolerances. 


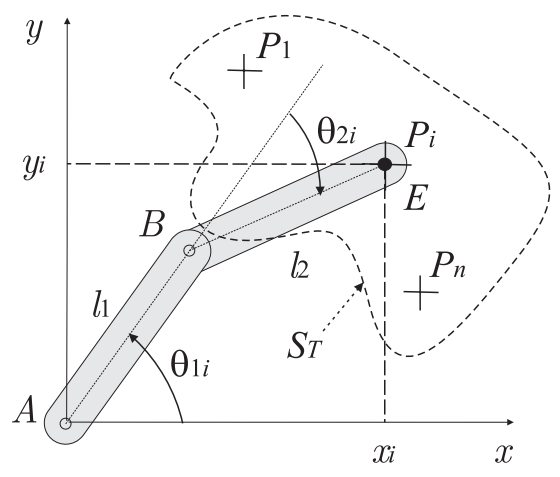

Figure 9. A $2 R$ Manipulator and its target $S_{T}$

5.1.1 Dimensioning of the $2 R$ manipulator Let $S_{T}$ be defined as a set of $n$ points $P_{1}, P_{2}, \cdots, P_{n}$. First, $E$ can hit all points in $S_{T}$ if and only if $l_{1}$ and $l_{2}$ satisfy the following conditions:

$$
\left\{\begin{array}{l}
\left|l_{1}-l_{2}\right| \leq r \\
l_{1}+l_{2} \geq R
\end{array}\right.
$$

with $r=\min _{i} d\left(A, P_{i}\right), \quad R=\min _{i} d\left(A, P_{i}\right), i=1, \cdots, n$ where $d\left(A, P_{i}\right)$ is the distance between $P_{i}$ and $A$. These conditions bound the feasible design variables space as shown in Fig.10.

The formulation of a robust design problem was given in section 2. For the manipulator under study, the set of design variables, $\mathbf{x}$, and the set of performance functions, $\mathbf{f}$, are given by eqs. (7),8).

$$
\begin{aligned}
\mathbf{x} & =\left[\begin{array}{ll}
l_{1} & l_{2}
\end{array}\right]^{T}, \mathbf{f}=\left[\mathbf{e}_{1}^{T} \cdots \mathbf{e}_{i}^{T} \cdots \mathbf{e}_{n}^{T}\right]^{T} \\
\mathbf{e}_{i} & =l_{1}\left[\begin{array}{ll}
C_{\theta_{1 i}} & S_{\theta_{1 i}}
\end{array}\right]^{T}+l_{2}\left[C_{\theta_{1 i}+\theta_{2 i}} S_{\theta_{1 i}+\theta_{2 i}}\right]^{T}
\end{aligned}
$$

where $\mathbf{e}_{i}$ is the vector of the Cartesian coordinates of $E$ at $P_{i}$. $C_{\theta_{j i}}=\cos \theta_{j i}, S_{\theta_{j i}}=\sin \theta_{j i}$ where $\theta_{j i}$ is the $j^{\text {th }}$ actuated joint variable at $P_{i}, j=1,2$.

The relation between the positioning error of $E$ at $P_{i}, \delta \mathbf{f}_{i}$, and dimensional variations $\delta l_{1}$, and $\delta l_{2}$, follows from eq.(8) and is given by eq.(9).

$$
\delta \mathbf{f}_{i}=\mathbf{J}_{x_{i}} \delta \mathbf{x} \quad \text { with } \quad \mathbf{J}_{x_{i}}=\left[\begin{array}{cc}
C_{\theta_{1 i}} & C_{\theta_{1 i}+\theta_{2 i}} \\
S_{\theta_{1 i}} & S_{\theta_{1 i}+\theta_{2 i}}
\end{array}\right] ; \delta \mathbf{x}=\left[\begin{array}{c}
\delta l_{1} \\
\delta l_{2}
\end{array}\right]
$$

The norm of $\delta \mathbf{f}=\left[\delta \mathbf{f}_{1}^{T}, \cdots, \delta \mathbf{f}_{i}^{T}, \cdots, \delta \mathbf{f}_{n}^{T}\right]^{T},\|\delta \mathbf{f}\|$, is the global positioning error of $E$ on $S_{T}$. The sensitivity Jacobian matrix of the manipulator, $\mathbf{J}_{x}$, is a $(2 n \times 2)$ matrix composed of matrices
$\mathbf{J}_{x_{i}}$. The relation between $\delta \mathbf{f}, \mathbf{J}_{x}$ and dimensional variations, $\delta \mathbf{x}$, is given by eq. 10$)$.

$$
\delta \mathbf{f}=\mathbf{J}_{x} \delta \mathbf{x} \quad \text { with } \quad \mathbf{J}_{x}=\left[\mathbf{J}_{x_{1}}^{T} \cdots \mathbf{J}_{x_{i}}^{T} \cdots \mathbf{J}_{x_{n}}^{T}\right]^{T}
$$

The robustness of the manipulator with respect to dimensional variations is quantified by robustness index $R I_{2}$, defined in section 3. $R I_{2}$ is the maximum singular value of $\mathbf{J}_{x}$ and corresponds to the maximum norm of positioning error of $E$, ||$\delta \mathbf{f}||_{\max }$, when the norm of dimensional variations is unitary, i.e.: $\delta l_{1}^{2}+\delta l_{2}^{2}=1$.

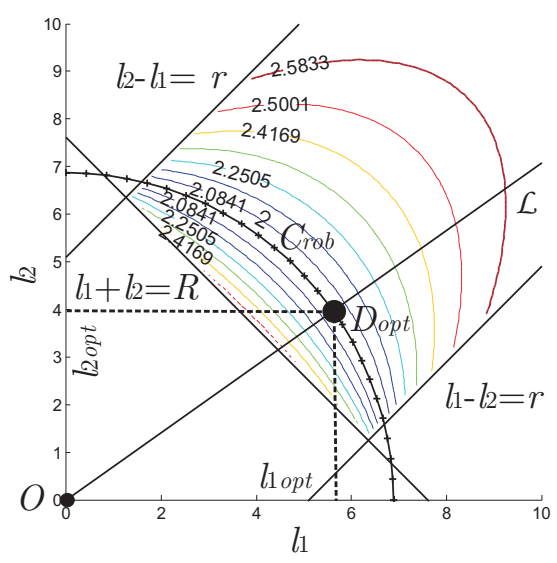

Figure 10. $R I_{2}=f\left(l_{1}, l_{2}\right)$

Let $S_{T}$ be made up of four points, $P_{1}, P_{2}, P_{3}, P_{4}$, whose Cartesian coordinates are $(1,5),(2,7),(3,7),(4,6)$, respectively. Fig.10 shows the isocontours of $R I_{2}$ in the feasible design variable space. We can notice that $R I_{2}$ isocontours form a family of ellipses and that $R I_{2}$ is a minimum when design variables belong to the circle $C_{r o b}$. In fact, the algebraic expression of $R I_{2}$ can be derived as shown in eq.(11):

$$
R I_{2}=\sqrt{n+\left|\sum_{i=1}^{n} \cos \theta_{2 i}\right|}=\sqrt{n+\left|\sum_{i=1}^{n} \frac{x_{i}^{2}+y_{i}^{2}-l_{1}^{2}-l_{2}^{2}}{2 l_{1} l_{2}}\right|}
$$

where $x_{i}$ and $y_{i}$ are the Cartesian coordinates of point $P_{i}$. Thus, the set of solutions $\left(l_{1}, l_{2}\right)$, satisfying eq. (11) for a fixed $R I_{2}$, is either ellipse $\varepsilon_{1}$ or ellipse $\varepsilon_{2}$ whose equations are $L_{1}^{2} / a_{1}^{2}+L_{2}^{2} / b_{1}^{2}=$ $c$ and $L_{1}^{2} / a_{2}^{2}+L_{2}^{2} / b_{2}^{2}=c$, respectively, where $a_{1}=b_{2}=1 / R I_{2}$, $a_{2}=b_{1}=1 / \sqrt{2 n-R I_{2}^{2}}$. $L_{1}$ and $L_{2}$ are the expressions of $l_{1}$ and $l_{2}$ in the coordinate frame rotated of $45 \mathrm{deg}$ with respect to the 
reference frame of the design variable space. Thus, $\varepsilon_{1}$ and $\varepsilon_{2}$, depicted in Fig.11, are the isocontours of robustness index $R I_{2}$.

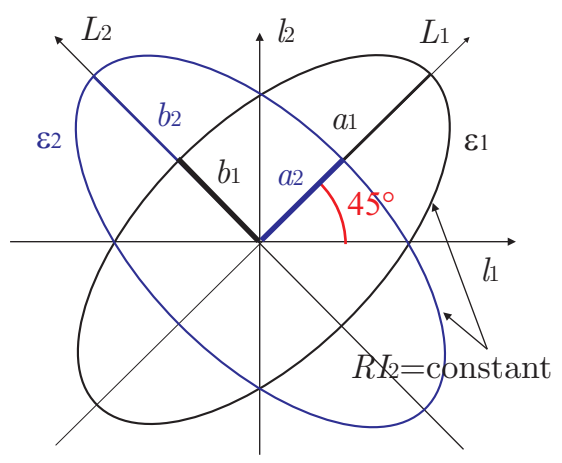

Figure 11. Design Variables $\left(l_{1}, l_{2}\right)$ corresponding to the same $R I_{2}$

$$
l_{1}^{2}+l_{2}^{2}=\frac{1}{n} \sum_{i=1}^{n} x_{i}^{2}+y_{i}^{2}=\frac{1}{n} \sum_{i=1}^{n} d^{2}\left(A, P_{i}\right)
$$

According to eq.(11), $R I_{2}$ is a minimum when eq.(12) is satisfied, i.e.: when dimensioning $\left(l_{1}, l_{2}\right)$ belongs to the circle of radius the square root of the mean of square distances between points $A$ and $P_{i}$ and centered at the origin of the design space variable. Therefore, this circle corresponds to $C_{r o b}$. Its radius is equal to 6.87 . Thus, there exists an infinite number of dimensionings $\left(l_{1}, l_{2}\right)$ that minimize $R I_{2}$. Three of them are depicted in Fig. 12 .

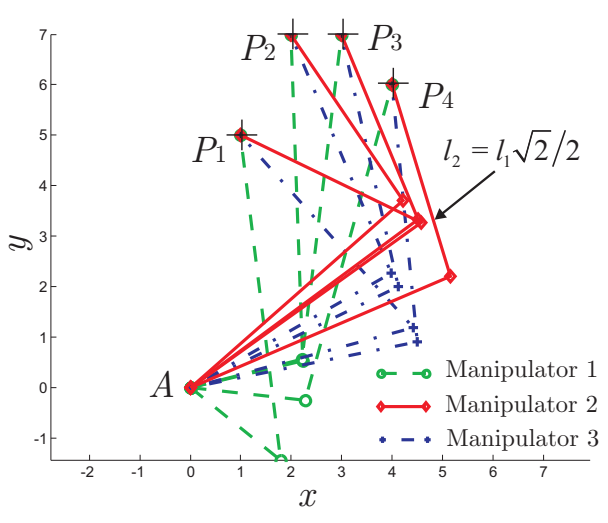

Figure 12. Robust Manipulators

According to eq. (11), the maximum global positioning error of $E$ is a minimum when cosines of angles $\theta_{2 i}$ tend towards zero. It means that the links of a robust $2 R$ manipulator should be almost perpendicular. That is apparent in Fig.12. The obtained robust dimensions are independent of the amount of variations and tolerate globally the largest variations.

As there are several robust manipulators, the designer can choose another criterion to be optimized. For instance, he can take into account the cost or the complexity of the mechanism. Here, the optimal robust manipulator is supposed to be the one with the best dexterity. This criterion is frequently used in manipulator design. It evaluates the ease of a manipulator to execute motions or arbitrary motions in all directions. It is quantified by the condition number of its kinematic Jacobian matrix, [20]. The smaller this condition number, the higher the dexterity. Besides, the manipulator is isotropic when its condition number is equal to one, [20]. Let $\mathbf{J}_{k}$ be the kinematic Jacobian matrix of the $2 R$ manipulator:

$$
\mathbf{J}_{k}=\left[\begin{array}{cc}
-l_{1} \sin \left(\theta_{1}\right)-l_{2} \sin \left(\theta_{1}+\theta_{2}\right) & -l_{2} \sin \left(\theta_{1}+\theta_{2}\right) \\
l_{1} \cos \left(\theta_{1}\right)+l_{2} \cos \left(\theta_{1}+\theta_{2}\right) & l_{2} \cos \left(\theta_{1}+\theta_{2}\right)
\end{array}\right]
$$

For any posture of the manipulator defined by angle $\theta_{2}$, the condition number of $\mathbf{J}_{k}$ is a minimum if and only if $l_{2}=l_{1} \sqrt{2} / 2$ [20]. Let $\mathcal{L}$ be the line of equation $l_{2}=l_{1} \sqrt{2} / 2$. $D_{\text {opt }}$, the intersection of $C_{\text {rob }}$ with $\mathcal{L}$, depicts the optimal robust manipulator, cf Fig.10. In conclusion, the $2 R$ manipulator of link lengths $l_{1}=l_{1 o p t}=5.61$ and $l_{2}=l_{2 o p t}=3.97$ is the optimal robust one, i.e.: the one with the best dexterity among the least sensitive ones to dimensional variations that allow their end effector $E$ to hit all points in $S_{T}$. This manipulator is the second one depicted in Fig.12, i.e.: the one whose links are depicted with bold lines.

In conclusion, the robust design method gave the set of all the robust manipulators. The optimal dimensioning was chosen among this set by means of another criterion, namely the dexterity. In the following section, the tolerance synthesis method presented in section 4 is used to compute the optimal dimensional tolerances of the selected manipulator.

\subsubsection{Tolerance synthesis of the $2 R$ manipulator} In addition to the fact that $E$ has to hit every point of $S_{T}$, its positioning error has to be smaller than $10 \mu \mathrm{m}$ whatever its pose.

Fig.13 depicts the design sensitivity ellipses of the optimal robust manipulator defined in the previous section, when target $S_{T}$ is defined by $P_{1}, P_{2}, P_{3}, P_{4}$, plotted in Fig.12. The shape, size and orientation of these ellipses depend only on the second actuated joint variable, $\theta_{2}$, of the manipulator. $\theta_{2}$ belongs to interval $\left[\theta_{2 \min }, \theta_{2 \text { max }}\right]$ to allow $E$ to hit all points of $S_{T}$. Moreover, points on these ellipses lead to the same positioning error of $E$, equal to $10 \mu \mathrm{m}$.

The optimal dimensional tolerances $\Delta l_{1 o p t}$ and $\Delta l_{2 o p t}$ of lengths $l_{1}$ and $l_{2}$ are computed from the tolerance synthesis 


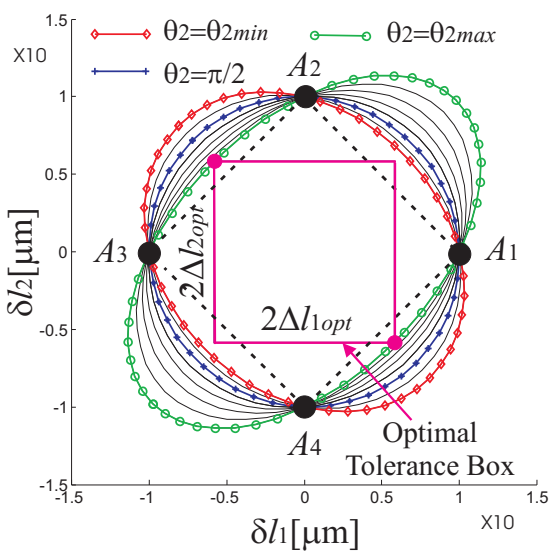

Figure 13. Tolerance Box

method developed in section 4 . The ellipse used in the tolerance synthesis algorithm is the one corresponding to $\theta_{2 \max }$, i.e.: the one with the smallest semi-axis. Finally, $\Delta l_{1 \text { opt }}=$ $\Delta l_{2 o p t}=5.82 \mu \mathrm{m}$ and the corresponding tolerance box is depicted in Fig.13.

Points $A_{1}(1,0), A_{2}(0,1), A_{3}(-1,0), A_{4}(0,-1)$ belong to all design sensitivity ellipses of the manipulator because a unitary variation in $l_{i}$ and no variation in $l_{j},(i \neq j)$, lead to a unitary positioning error of its end effector. As an ellipse is convex, square $A_{1} A_{2} A_{3} A_{4}$ is included in all the design sensitivity ellipses whatever the bounds of $\theta_{2}$. It follows that eq.(14) is a sufficient condition for the positioning error of $E$ to be smaller than $10 \mu \mathrm{m}$ whatever its pose.

$$
\Delta l_{1}+\Delta l_{2} \leq 10 \mu \mathrm{m}
$$

Without the tolerance synthesis method developed in section 7, the designer would have chosen dimensional tolerances $\Delta l_{1}$ and $\Delta l_{2}$ by means of eq.(14). Here, $\Delta l_{1 o p t}$ and $\Delta l_{2 o p t}$ do not respect eq.(14) because $\Delta l_{1 \text { opt }}+\Delta l_{2 o p t}=11.64 \mu \mathrm{m}$. However, they allow the positioning error of $E$ to be smaller that $10 \mu \mathrm{m}$ on $S_{T}$. So, knowing the target of the manipulator, the tolerance synthesis method proposed in section 4 is more interesting than the sufficient condition, defined by eq.(14), to synthesize its dimensional tolerances.

The $2 R$ manipulator has been studied in order to get graphical interpretations of the results and algebraic expressions of robustness index $R I_{2}$. However, the foregoing methods can be applied to more general mechanisms and $R I_{2}$ may be computed numerically, as for the study of the $3 R$ manipulator in the following section.

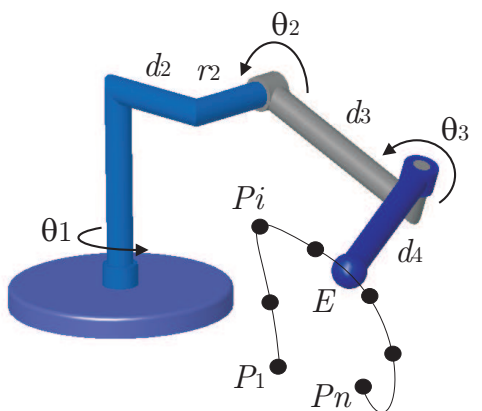

Figure 14. $3 R$ Manipulator

\subsection{Tolerance synthesis of a $3 R$ manipulator}

A three-dof serial positioning manipulator with three revolute joints is shown in Fig.14. Modified D-H parameters are used to describe its geometry [21]. $\theta_{1}, \theta_{2}, \theta_{3}$ are the actuated joint variables of the $3 R$ manipulator and $d_{2}, r_{2}, d_{3}, d_{4}$ denote its dimensions. Its inverse geometric model was studied in [22]. The positioning error, $\varepsilon_{E}$, of end effector $E$, has to be smaller than $10 \mu \mathrm{m}$ at any point $P_{i}, i=1, \cdots, n$, of a path. Variations in $\theta_{1}, \theta_{2}$ and $\theta_{3}$ are negligible because the encoders are supposed to be very accurate. So, $\varepsilon_{E}$ depends on variations in the other D-H parameters. Here, only variations in $d_{2}, r_{2}, d_{3}$ are considered in order to get graphical representation.

The relation between the positioning error of $E$ at $P_{i}, \delta \mathbf{f}_{i}$, and dimensional variations $\delta d_{2}, \delta r_{2}$, and $\delta d_{3}$, is given by eq. 15 .

$$
\delta \mathbf{f}_{i}=\mathbf{J}_{x_{i}} \delta \mathbf{x} \quad \text { with } \quad \mathbf{J}_{x_{i}}=\left[\begin{array}{ccc}
\cos \theta_{1 i} & -\sin \theta_{1 i} & \cos \theta_{1 i} \cos \theta_{2 i} \\
\sin \theta_{1 i} & \cos \theta_{1 i} & \sin \theta_{1 i} \cos \theta_{2 i} \\
0 & 0 & -\sin \theta_{2 i}
\end{array}\right]
$$

where $\theta_{1 i}$ and $\theta_{2 i}$ are the values of $\theta_{1}$ and $\theta_{2}$ at $P_{i}$, computed with the inverse kinematic model of the manipulator [22]. $\mathbf{f}=$ $\left[\begin{array}{lll}e_{x} & e_{y} & e_{z}\end{array}\right]^{T}$ where $e_{x}, e_{y}$ and $e_{z}$ denote the Cartesian coordinates of $E$ and $\delta \mathbf{x}=\left[\begin{array}{lll}\delta d_{2} & \delta r_{2} & \delta d_{3}\end{array}\right]^{T}$ where $\delta d_{2}, \delta r_{2}, \delta d_{3}$ are variations in $d_{2}, r_{2}, d_{3}$, respectively.

The norm of $\delta \mathbf{f}=\left[\delta \mathbf{f}_{1}^{T}, \cdots, \delta \mathbf{f}_{i}^{T}, \cdots, \delta \mathbf{f}_{n}^{T}\right]^{T},\|\delta \mathbf{f}\|$, is the global positioning error of $E$. The sensitivity Jacobian matrix of the manipulator, $\mathbf{J}_{x}$, is a $(3 n \times 2)$ matrix composed of matrices $\mathbf{J}_{x_{i}}$. The relation between $\delta \mathbf{f}, \mathbf{J}_{x}$, and $\delta \mathbf{x}$, is given by eq. (10).

Assuming that $n=5$ and Cartesian coordinates of points $P_{1}, P_{2}, P_{3}, P_{4}, P_{5}$, are $(1,1,1),(2,-2,3),(5,6,2),(-1,-4,3),(2,3$, $5)$, respectively. Index $R I_{2}$, defined in section 3 , is used to find the robust dimensioning of the manipulator, and is computed numerically. Here, $R I_{2}$ is a minimum and the design of the manipulator is robust when $d_{2}=1.75, r_{2}=2.5, d_{3}=3.25$, and $d_{4}=2.5$.

Fig. 15 depicts the most restrictive ellipsoid of the manipulator and its optimal tolerance box. The most restrictive ellipsoid, 


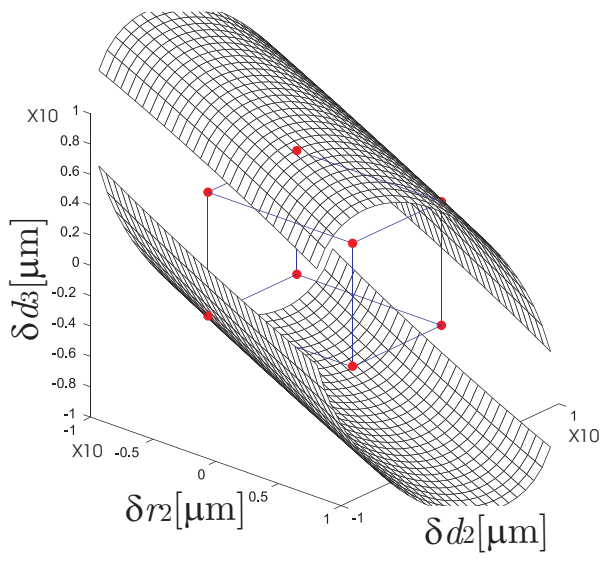

Figure 15. The Most Restrictive Ellipsoid \& the Optimal Tolerance Box

$\xi_{m r}$, is the one with the smallest semi-axis among the five design ellipsoids of the manipulator and corresponds to point $P_{1}$. The tolerance synthesis method proposed in section 7 is used to compute the optimal tolerance box included in the most restrictive ellipsoid, i.e.: the following algorithm is used to compute $\Delta d_{2 o p t}, \Delta r_{2 o p t}$ and $\Delta d_{3 o p t}$ :

$$
\begin{cases}\max _{\mathbf{u}} & \left|u_{1} u_{2} u_{3}\right| \\ \text { s.t. } & U\left(u_{1}, u_{2}, u_{3}\right) \in \xi_{m r} \\ & u_{1} \geq 0 \\ & u_{3} \geq 0 \\ & \left|u_{i}\right| \geq \Delta x_{\text {imin }}, i=1, \cdots, 3\end{cases}
$$

where $\Delta x_{1 \text { min }}=1 \mu \mathrm{m}, \Delta x_{2 \min }=\frac{r_{2}}{d_{2}} \Delta x_{1 \text { min }}, \Delta x_{3 \min }=\frac{d_{3}}{d_{2}} \Delta x_{1 \text { min }}$

The results of this optimization problem are: $u_{1}=4.08 \mu$, $u_{2}=-5.77 \mu$ and $u_{3}=4.08 \mu$. Thus, $\Delta d_{2 o p t}=4.08 \mu \mathrm{m}, \Delta r_{2 o p t}=$ $5.77 \mu \mathrm{m}$ and $\Delta d_{3 o p t}=4.08 \mu \mathrm{m}$.

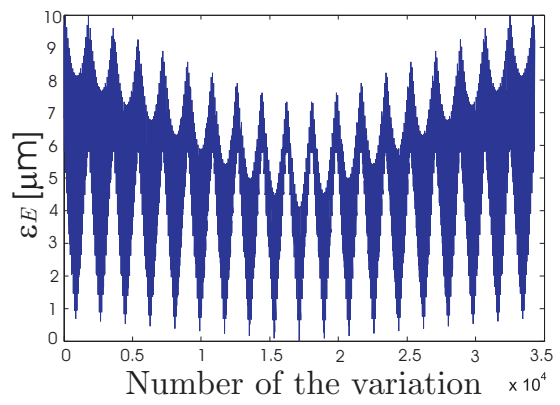

Figure 16. Validation of the Optimal Tolerance Box

Fig. 16 depicts the values of $\varepsilon_{E}$ when $\delta d_{2}, \delta r_{2}, \delta d_{3}$, are be- tween $-\Delta d_{2 o p t}$ and $\Delta d_{2 o p t},-\Delta r_{2 o p t}$ and $\Delta r_{2 o p t},-\Delta d_{3 o p t}$ and $\Delta d_{3 o p t}$, respectively, and for the five poses of the manipulator . $\varepsilon_{E}$ is always smaller than $10 \mu \mathrm{m}$. It means that the positioning error of $E$ is smaller than $10 \mu \mathrm{m}$ for any posture of the manipulator when the tolerances of $d_{2}, r_{2}$, and $d_{3}$ are $\Delta d_{2 o p t}, \Delta r_{2 o p t}$, and $\Delta d_{3 o p t}$, respectively.

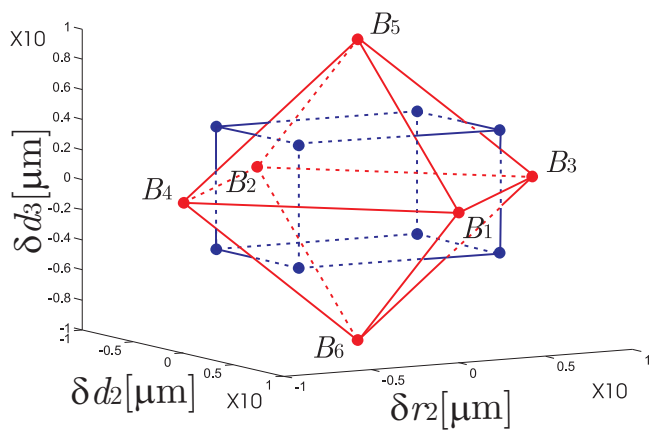

Figure 17. The Optimal Tolerance Box is not included in the Octahedron

Points $B_{1}(1,0,0), B_{2}(-1,0,0), B_{3}(0,1,0), B_{4}(0,-1,0)$, $B_{5}(0,0,1)$ and $B_{6}(0,0,-1)$ belong to all design sensitivity ellipsoids of the manipulator because a unitary variation of a design variable and no variation of the others lead to a unitary variation of the position of $E$. As an ellipsoid is a convex volume, octahedron $B_{1}, B_{2}, B_{3}, B_{4}, B_{5}, B_{6}$ depicted in Fig. 17 is included in all the design sensitivity ellipsoids of the manipulator such that a point on the surface leads to a positioning error of $E$ equal to $10 \mu \mathrm{m}$. It follows that eq.(16) is a sufficient condition for the positioning error of $E$ to be smaller than $10 \mu \mathrm{m}$ whatever its pose.

$$
\Delta d_{2}+\Delta r_{2}+\Delta d_{3} \leq 10 \mu \mathrm{m}
$$

Without the tolerance synthesis method proposed in section 4. the designer would have chosen dimensional tolerances $\Delta d_{2}$, $\Delta r_{2}$, and $\Delta d_{3}$ by means of eq.(16). Here, $\Delta d_{2 o p t}, \Delta r_{2 o p t}$, and $\Delta d_{3 o p t}$ do not respect eq.(16) because $\Delta d_{2 o p t}+\Delta r_{2 o p t}+\Delta d_{3 o p t}=$ $13.9 \mu \mathrm{m}$. It means that the optimal tolerance box is not included in octahedron $B_{1}, B_{2}, B_{3}, B_{4}, B_{5}, B_{6}$, as depicted by Fig. 17. However, they allow the positioning error of $E$ to be smaller that 10 $\mu \mathrm{m}$ at each pose $P_{i}, i=1, \cdots, 5$. So, knowing the target of the manipulator, the tolerance synthesis method proposed in section 4 is more interesting than the sufficient condition, defined by eq.(16), to synthesize its dimensional tolerances.

In conclusion, the optimal dimensional tolerances of the $3 R$ manipulator are $\Delta d_{2}=4.08 \mu \mathrm{m}, \Delta r_{2}=5.77 \mu \mathrm{m}$ and, $\Delta d_{3}=$ $4.08 \mu \mathrm{m}$ so that the positioning error of $E$ is less than $10 \mu \mathrm{m}$ at any point $P_{i}, i=1, \cdots, 5$. 


\section{Conclusions}

This paper has provided a new and efficient tolerance synthesis method for mechanisms, based on a robust design approach. The study of the robustness of a mechanism follows two consecutive steps, which are independent and complementary. The first step aims at computing its robust dimensions by means of an appropriate robustness index. The Euclidean norm of the sensitivity Jacobian matrix is such an index. The study of a damper confirmed that the Euclidean norm of its sensitivity Jacobian matrix is more suitable than its condition number, to quantify the robustness of a mechanism. This method yields the set of all the robust manipulators and allows the designer to integrate other criteria. Then, the developed tolerance synthesis method is used to compute the optimal tolerance box of the selected robust manipulator. The theory is illustrated by two serial manipulators. The application of this theory to the robust design and tolerance synthesis of parallel manipulators is one of the next steps in our research work.

\section{REFERENCES}

[1] Khalil, W., Besnard, S., Lemoine, P., Comparison study of the geometric parameters calibration methods, International Journal of Robotics and Automation, Vol.15, 2000, 56-67.

[2] Taguchi, G., On robust technology development, Bringing Quality Engineering Upstream, ASME Press , 1993.

[3] Kalsi, M., Hacker, K., Lewis, K., A comprehensive robust design approach for decision trade-offs in complex systems design, Transactions of the ASME, Journal of Mechanical Design, Vol.121, march, 2001, 1-10.

[4] Chen, W., Allen, J.K., Tsui, K-L., Mistree, F., A procedure for robust design: minimizing variations caused by noise factors and control factors, Transactions of the ASME, Journal of Mechanical Design, Vol.118, december, 1996, 478-485.

[5] Sundaresan, S., Ishii, K., Houser, D.R., A robust optimization procedure with variations on design variables and constraints, DE.Vol 65-1, Advances in Design Automation, ASME 1993, Vol.1, 1993, 379-386.

[6] Chase, K., Gao, J., Magleby, S.P., Sorensen, C.D., Including geometric feature variations in tolerance analysis of mechanical assemblies, IIE transactions, Vol.28, 1996, 795-807.

[7] Gao, J., Chase, K., Magleby, S.P., Generalized 3-D tolerance analysis of mechanical assemblies with small kinematic adjustments, IIE transactions, Vol.30, 1998, 367-377.

[8] Parkinson, D.B., The Application of a Robust Design Method to Tolerancing, Transactions of the ASME, Journal of Mechanical Design, Vol.122, june, 2000, 149-154.

[9] Rajagopalan, S., Cutkosky, M., Error analysis for the InSitu fabrication of mechanisms, Transactions of the ASME,
Journal of Mechanical Design, Vol.125, december, 2003, 809-822.

[10] Zhang, C., Wang, B., Robust design of assembly design and machining tolerance allocations, IIE Transactions 30(1), 1998, 17-29.

[11] Lee, W.J., Woo, T.C., Chou, S.Y. Tolerance synthesis for nonlinear systems based on nonlinear programming, IIE Transactions 25(1), 1993, 51-61.

[12] Gadallah, M., ElMaraghy, H., The tolerance optimization problem using a system of experimental design, In Advances in Design Automation, Vol.69, ASME Publishers, NY, 1994, 251-265.

[13] Zhu, J., Ting, K.L., Performance distribution analysis and robust design, Transactions of the ASME, Journal of Mechanical Design, Vol.123, march, 2001, 11-17.

[14] Zhang, G., Porchet, M., Some new developments in tolerance design in CAD, DE-Vol.66-2, Advances in Design Automation - Vol.2, ASME, 1993.

[15] Al-widyan, K., Angeles, J., A model-based framework for robust design, Recent Advances in Integrated Design and Manufacturing in Mechanical Engineering, Kluwer Academic Publisher, 2003.

[16] Ting, K.L., Long, Y., Performance quality and tolerance sensitivity of mechanisms, Transactions of the ASME, Journal of Mechanical Design, Vol.118, march, 1996, 144-150.

[17] Hu, S.J., Webbink, R., Lee, J., Long, Y., Robustness evaluation for compliant assembly systems, Transactions of the ASME, Journal of Mechanical Design, Vol.125, June, 2003, 262-267.

[18] Caro, S., Bennis, F., Wenger, P., Search for an optimal robustess index for the design of mechanisms, Technical Report, RI 2002-16, IRCCyN, Ecole Centrale de Nantes, July 2002.

[19] Parkinson, A., Robust mechanical design using engineering models, Transactions of the ASME, Journal of Mechanical Design, Vol.117, June, 1995, 48-54.

[20] Angeles, J., Fundamentals of Robotic Mechanical Systems, Springer-Verlag, New-York, ISBN 0-387-94540-7, 1997.

[21] Khalil, W., Kleinfinger, J.F. A new geometric notation for open and closed loop robots, Proc.IEEE Int. Conf. Rob. and Aut., 1986, 1174-1179.

[22] Wenger, P., Classification of $3 R$ positioning manipulators, Transactions of the ASME, Journal of Mechanical Design, Vol.120, june, 1998, 327-332. 